\begin{tabular}{|c|c|}
\hline Title & Effect of Substrate Bias on Production and Transport of Etchant Ions in a Magnetic Neutral Loop Discharge Plasma \\
\hline Author(s) & A sami, Y usuke; Sugawara, Hirotake \\
\hline Citation & $\begin{array}{l}\text { IEEE T ransactions on Plasma Science, 42(10), 2540-2541 } \\
\text { https://doi.org/10.1109/T PS.2014.2337753 }\end{array}$ \\
\hline Issue Date & $2014-10-21$ \\
\hline Doc URL & http:/hdl.handle.net/2115/57754 \\
\hline Rights & $\begin{array}{l}\text { (c) } 2014 \text { IEEE. Personal use of this material is permitted. Permission from IEEE must be obtained for all other uses, in } \\
\text { any current or future media, including reprinting/republishing this material for advertising or promotional purposes, } \\
\text { creating new collective works, for resale or redistribution to servers or lists, or reuse of any copyrighted component of } \\
\text { this work in other works. }\end{array}$ \\
\hline Tyре & article (author version) \\
\hline File Information & A sami-2014-huscap68030.pdf \\
\hline
\end{tabular}

Instructions for use 


\title{
Effect of Substrate Bias on Production and Transport of Etchant Ions in a Magnetic Neutral Loop Discharge Plasma ${ }^{1}$
}

\author{
Yusuke Asami and Hirotake Sugawara ${ }^{2}$
}

\begin{abstract}
Production and transport of etchant ions in an $\mathrm{rf}(13.56 \mathrm{MHz})$ magnetic neutral loop discharge plasma in the presence and the absence of an ac $(1.695 \mathrm{MHz})$ substrate bias were simulated using a Monte Carlo method. Under the bias, electrons accelerated near the neutral loop were attracted to the substrate and produced more etchant ions near the substrate than in the absence of the bias. Effect of bias leading to enhancement of the ion inflow to the substrate was discussed.
\end{abstract}

Etchant species in neutral loop (NL) discharge (NLD) plasma, a type of inductively coupled magnetized plasma $[1,2]$. are produced mainly around the NL of zero magnetic field and the separatrices of the quadrupole magnetic field applied to the NLD plasmas $[3,4]$. The transport of the etchant species is a key feature in control of the NLD plasma via magnetic field for uniform large-area processing $[4,5]$. This paper reports an effect of ac substrate bias preferable for enhancement of etchant ion inflow to the substrate at the bottom of the chamber. Production of $\mathrm{CF}_{3}^{+}$in a $\mathrm{CF}_{4} \mathrm{NLD}$ plasma and their inflow to the substrate are depicted.

The model NLD chamber was a cylinder with a 40-cm height and a 20-cm inner radius surrounded by three coaxial dc coils and a one-turn rf antenna $(13.56 \mathrm{MHz}, 25 \mathrm{~A}$ amplitude). A quadrupole magnetic field induced by the currents of the top, middle, and bottom coils (60-turn), +95.6, -115.5, and +95.6 A, respectively, had the NL at $r=11 \mathrm{~cm}$ and $z=0$, where $r=\sqrt{x^{2}+y^{2}}$. Electrons obtained energy near the NL and thus the NLD plasma became ring-shaped.

The simulation was performed in two steps. In step $1,5 \times 10^{4}$ electrons were traced using a Monte Carlo method to obtain time-averaged spatial distribution $P(r, z)$ of the ionization collisions producing $\mathrm{CF}_{3}^{+}$. The $\mathrm{CF}_{4}$ pressure was set at 5 mTorr. The electron collision cross sections [6] used in the simulation included dissociative processes to produce $\mathrm{CF}_{3}^{+}, \mathrm{CF}_{2}^{+}, \mathrm{CF}^{+}, \mathrm{F}^{+}, \mathrm{F}^{-}, \mathrm{CF}_{3}^{2+}, \mathrm{CF}_{2}^{2+}, \mathrm{CF}_{3}, \mathrm{CF}_{2}, \mathrm{CF}$, and $\mathrm{F}$. The electrons were released from the NL with a weight of $10^{5}$ and their trajectories in real space $(x, y, z)$ under electric and magnetic fields were calculated using a Runge-Kutta method with a time step $\Delta t=37 \mathrm{ps}$. The magnetic field had $B_{r}$ and $B_{z}$ components $\left(B_{\theta}=0\right)$, and the electric field had $E_{\theta}$ by the rf antenna and $E_{r}$ and $E_{z}$ due to the bias and space charge.

\footnotetext{
${ }^{1}$ Published source: IEEE Transactions on Plasma Science, Vol. 42, No. 10, pp. 2540-2541, October 2014. Manuscript received November 2, 2013; revised July 2, 2014; accepted July 8, 2014. Date of publication July 29, 2014; date of current version October 21, 2014. This work was supported in part by the Japan Society for the Promotion of Science under Grant 22540500 and Grant 25400528, and in part by ULVAC, Inc. Chigasaki, Kanagawa, Japan.. Digital Object Identifier 10.1109/TPS.2014.2337753

${ }^{2}$ The authors are with the Graduate School of Information Science and Technology, Hokkaido University, Sapporo 060-0814, Japan (e-mail: sugawara@ist.hokudai.ac.jp).
} 
The plasma density was about $10^{7}-10^{8} \mathrm{~cm}^{-3}$ around the NL. The electron reflectivity at the chamber wall was assumed to be $60 \%$. The charge on the wall was also taken into account in the calculation of the space charge field. The ac bias (100-V amplitude, 1.695 MHz, sinusoidal) was applied to the substrate. The positions of $\mathrm{CF}_{3}^{+}$production were sampled and mapped to the $r-z$ plane assuming azimuthal homogeneity in $\theta$, where $x=r \cos \theta$ and $y=r \sin \theta$.

In step 2, flight of $\mathrm{CF}_{3}^{+}$was traced to obtain the $\mathrm{CF}_{3}^{+}$inflow distribution $I(r)$ at the substrate in the same space charge fields as in step 1 . The number of $\mathrm{CF}_{3}^{+}$was $2.5 \times 10^{5}$ commonly in the absence and the presence of the substrate bias. The initial positions of $\mathrm{CF}_{3}^{+}$were chosen at random from $P(r, z)$, and they were traced for $2 \times 10^{4} \mathrm{rf}$ periods or until they reached the substrate, sidewall, or chamber ceiling. Only elastic collision with $\mathrm{CF}_{4}$ was considered as the in flight process of $\mathrm{CF}_{3}^{+}$for simplicity.

Fig. 1 shows $P(r, z)$ normalized in the $r$ - $z$ plane (the region of $P>20 \mathrm{~m}^{-2}$ is shown) and $I(r) . P(r, z)$ expanded toward the substrate along the downward separatrix under the bias. The bias attracted not only $\mathrm{CF}_{3}^{+}$during its negative phase, but also energetic electrons during the positive phase. The $\mathrm{CF}_{3}^{+}$production increased, and this was in part due to electron heating by the bias. Repulsion of electrons during the negative phase did not appear significantly because of the geometrical asymmetry between the biased substrate and chamber wall. Because the flight of ions was governed partly by the space charge field, the $\mathrm{CF}_{3}^{+}$production near the substrate contributed to the increase of the $\mathrm{CF}_{3}^{+}$ inflow to the substrate. In addition, $I(r)$ was also expanded from the foot of separatrix to a wider range on the substrate. Even in the presence of in-flight loss of $\mathrm{CF}_{3}^{+}$, for example by recombination and charge exchange not considered in the simulation, the $\mathrm{CF}_{3}^{+}$production near the substrate under bias would still be advantageous because the in-flight loss would be more significant for longer flight from the $\mathrm{CF}_{3}^{+}$source around the NL to the substrate in the absence of the bias.

A bias applied to the substrate in a $\mathrm{CF}_{4}$ NLD plasma attracted energetic electrons from the NL. As a result, the region of $\mathrm{CF}_{3}^{+}$etchant production approached the substrate. This tendency was considered to be preferable for the increase of the $\mathrm{CF}_{3}^{+}$inflow to the substrate.

\section{References}

[1] T. Uchida, "Application of radio-frequency discharged plasma produced in closed magnetic neutral line for plasma processing," Japan. J. Appl. Phys., vol. 33, no. 1A, pp. L43-L44, Jan. 1994.

[2] T. Uchida and S. Hamaguchi, "Magnetic neutral loop discharge (NLD) plasmas for surface processing," J. Phys. D, Appl. Phys., vol. 41, no. 8, pp. 083001-1-083001-21, Apr. 2008.

[3] T. Osaga, H. Sugawara, and Y. Sakurai, "Structure and dynamics of a magnetic neutral loop discharge plasma described using electron motion in quadrupole magnetic field," Plasma Sources Sci. Technol., vol. 20, no. 6, pp. 065003-1-065003-7, Nov. 2011.

[4] Y. Sakurai, T. Osaga, and H. Sugawara, "Control of magnetic field in neutral loop discharge plasma for uniform distribution of ion flux on substrate," IEEE Trans. Plasma Sci. vol. 39, No. 11, Pt. 1, 2550-2551, Nov. 2011. 


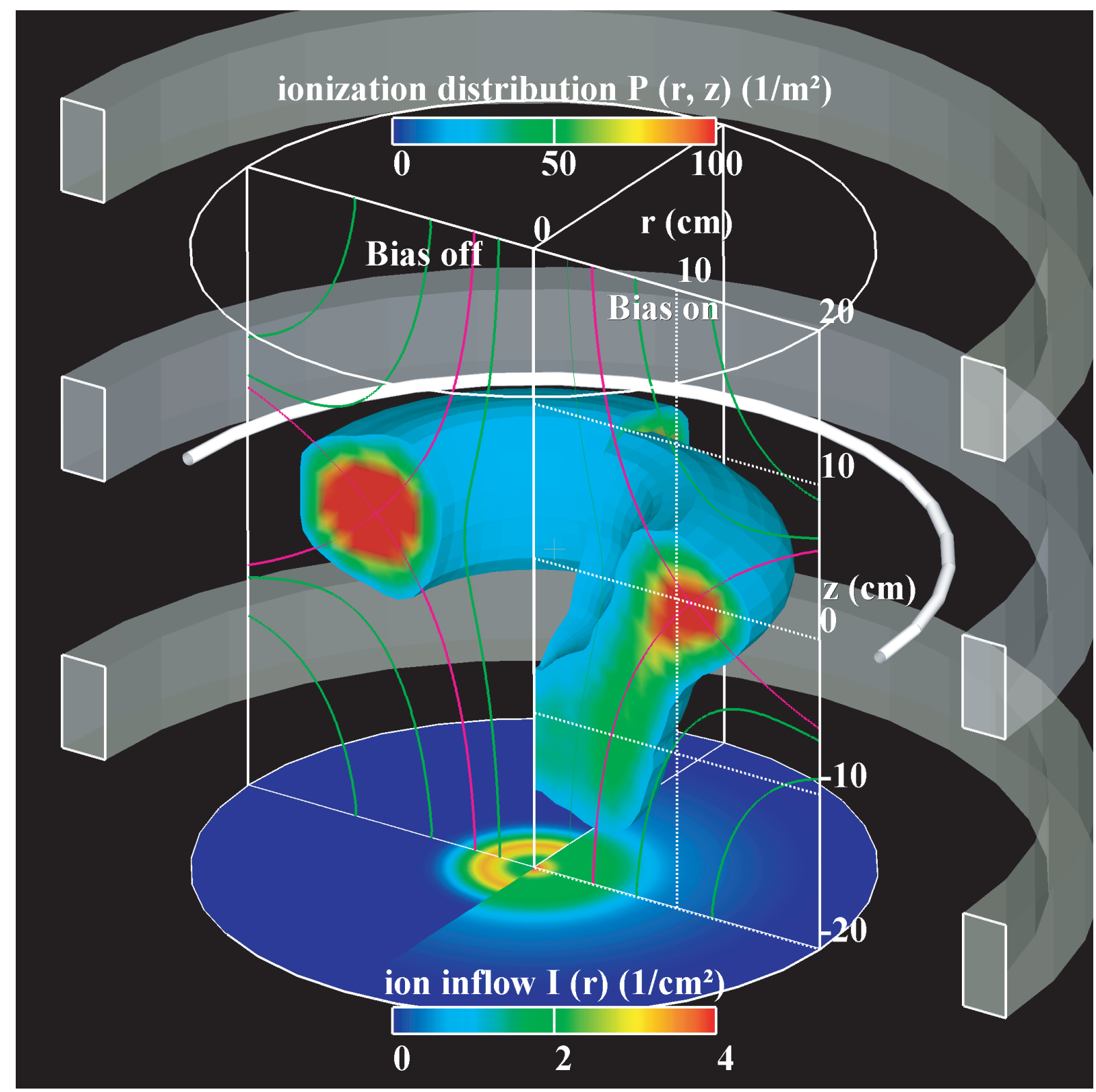

Figure 1: Spatial distribution of dissociative ionization collisions, $P(r, z)$, producing $\mathrm{CF}_{3}^{+}$ and ion inflow distribution, $I(r)$, on substrate in the absence (left) and the presence (right) of substrate bias of $100 \mathrm{~V}$. The green curves represent the magnetic field lines, the pink ones are separatrices of the quadrupole magnetic field, and the NL is at the cross point of the separatrices. The bias attracted the ion production region from the NL toward the substrate along the downward separatrix. The ion inflow to the substrate spread and increased under the bias.

[5] T. Yoshida, Y. Sakurai, H. Sugawara, and A. Murayama, "Control of magnetic field for sustainment of ion production and uniform ion flux to substrate in neutral loop discharge plasma," Electrical Eng. in Japan, vol. 181, no. 1, pp. 9-16, Oct. 2013.

[6] M. Kurihara, Z. Lj. Petrović, and T. Makabe, "Transport coefficients and scattering cross-sections for plasma modelling in $\mathrm{CF}_{4}$-Ar mixtures: a swarm analysis," J. Phys. D, Appl. Phys., vol. 33, no. 17, pp. 2146-2153, Sep. 2000. 